\title{
Unmet need and unintended pregnancy: The bigger picture
}

Ian Askew

Population Council

Follow this and additional works at: https://knowledgecommons.popcouncil.org/departments_sbsr-rh

Part of the Demography, Population, and Ecology Commons, Family, Life Course, and Society Commons, Gender and Sexuality Commons, International Public Health Commons, Maternal and Child Health Commons, and the Women's Health Commons How does access to this work benefit you? Let us know!

\section{Recommended Citation}

Askew, lan. 2013. "Unmet need and unintended pregnancy: The bigger picture," IUSSP Working Paper. Nairobi: Population Council. 


\title{
Unmet Need and Unintended Pregnancy:
}

\section{The Bigger Picture}

\author{
Ian Askew \\ Co-Director, Strengthening Evidence for Programming on Unintended Pregnancy \\ (STEP UP), Population Council, Nairobi, Kenya
}

Is Access Enough? Understanding and addressing unmet need in family planning Nanyuki, Kenya, 14 - 17 May 2013

International Union for the Scientific Study of Population (IUSSP)

Scientific Panel on Reproductive Health

and

Strengthening Evidence for Programming on Unintended Pregnancy (STEP UP) Consortium

and

African Institute for Development Policy (AFIDEP) 


\begin{abstract}
Prevention of unintended pregnancy is often considered the key outcome of reducing unmet need; it is the basic right of women and couples to become pregnant only when intended. This paper explores this relationship by reviewing the definition and measurement of unintended pregnancy, identifying key trends in its distribution within and between populations, and analysing the relationship between unintended pregnancy, demand for FP and FP program performance. The determinants and consequences of unintended pregnancy are reviewed to further understand the implications of using it as a policy and programming goal. The paper concludes by reviewing the broader policy context in which unintended pregnancy and unmet need are used and identifies important considerations for the future.
\end{abstract}




\section{Why is unintended pregnancy important now?}

"The concept of unintended pregnancy has been essential to demographers in seeking to understand fertility, to public health practitioners in preventing unwanted childbearing and to both groups in promoting a woman's ability to determine whether and when to have children. Accurate measurement of pregnancy intentions is important in understanding fertility-related behaviors, forecasting fertility, estimating unmet need for contraception, understanding the impact of pregnancy intentions on maternal and child health, designing family planning programs and evaluating their effectiveness, and creating and evaluating community-based programs that prevent unintended pregnancy." (Santelli et al 2003: 94).

In addition to these myriad reasons, policy statements of most of the major international organizations engaged in family planning explicitly target the prevention of unintended pregnancy as the rationale for their investments in FP programming (see also Malarcher et al (2010: 178-179) for further justification for focusing on unintended pregnancy). For example: WHO's Reproductive Health Strategy (WHO 2004) and UNFPA's 'State of World Population 2012' report (UNFPA 2012) repeatedly mention the adverse outcomes associated with, and the need to reduce, unintended pregnancy; the UK's 'Framework for Results for Improving Reproductive, Maternal and Newborn Health' has two strategic priorities, one of which is to "prevent unintended pregnancies by enabling women and adolescent girls to choose whether, when and how many children they have" (DFID 2010: 2); and one of USAID's core development objectives is to "prevent 54 million unintended pregnancies" (USAID 2011: 6).

Over the past decade, however, and particularly since the London Summit on Family Planning in July 2012, policy statements, investment decisions and programming strategies for these same organizations have also focused on reducing unmet need for FP as a key goal, either in and of itself or as an intermediary to reducing unintended pregnancy. This paper considers the challenges and opportunities of simultaneously pursuing policy goals of reducing unintended pregnancy and reducing unmet need by revitalizing an understanding and appreciation of the concept and measurement of unintended pregnancy. The paper also reviews the implications of applying unintended pregnancy as a policy goal, both independently and in association with the goal of reducing unmet need. The paper reviews the broader set of determinants and consequences of unintended pregnancy and their implications for policy goals and programming investments. 


\section{What is an unintended pregnancy?}

A pregnancy is usually defined as unintended if it is either unwanted (i.e. occurred when no children or no more children were desired), or is mistimed at the time of conception (i.e. occurred earlier than desired but would have been desired at a later time). Conversely, an intended pregnancy is defined as occurring at the "right" time (as subjectively conceptualized by the woman) or later than desired (Santelli et al 2003; Santelli et al 2009). This definition was first developed by the U.S. National Survey of Family Growth (NSFG) in 1973 and subsequently adapted by the Demographic and Health Surveys (DHS) for use in developing countries; both definitions result in a pregnancy being categorised in one of three ways: intended, unwanted or mistimed.

These surveys ask women who have given birth to retrospectively describe their pregnancy intentions by combining their recollections of the desire for (more) children and their preferred timing of a pregnancy at the time of conception. Consequently, this methodology only measures the intendedness of pregnancies that were carried to term, that is, considered to be unplanned births (i.e. unwanted or mistimed); women are not asked about their intentions for a pregnancy that was terminated or ended in a miscarriage.

Calculations of the total number of unintended pregnancies in a population include, therefore, three factors:: i) all unplanned births; ii) all abortions (it is assumed that an abortion is always the result of an unintended pregnancy); and iii) all miscarriages from unintended pregnancies (it is assumed that intended and unintended pregnancies have the same probability of ending in miscarriage) (Singh et al 2010: 243).

Over the past 15 years there have been sustained discussions and debates about the conceptualization and definition of unintended pregnancy, mostly in the USA and other developed countries (see Santelli et al (2003) and Tsui et al (2010) for systematic literature reviews that discuss many of the key concepts). For developing countries, however, there have been very few analyses of unintended pregnancy, either in terms of its conceptualization, definition, measurement, or its determinants and consequences; no systematic review of this literature has been undertaken, although several analyses are available (e.g. Malarcher et al 2010; Exavery et al 2013; Stephenson et al 2008; Koenig et al 2006; Cu Le et al 2004; Marston \& Cleland 2003; Williams \& Sobieszczyk 2003). From the perspective of the analyses undertaken and ensuing discussions about unintended pregnancy in developed countries, several issues have emerged that should be considered 
when trying to understand the meaning of unintended pregnancy in developing country settings and the implications for women's lives and for FP/RH programming. These can be summarized as follows.

- A conceptual distinction between 'intendedness' and 'wantedness' has emerged: "Intendedness has to do with cognition and, specifically, with the degree of prior planning for a pregnancy, whereas wantedness has to do with affect and, specifically, with the feelings - both positive and negative - that occur in response to a pregnancy" (David 2011: 185). In developed country settings, a woman's ability to make this distinction appears to be strong enough to suggest that measures of pregnancy intendedness should include both cognitive and affective dimensions. Moreover, the degree to which a woman expresses her desire or happiness to be pregnant may influence outcomes of the pregnancy more than whether the pregnancy was planned.

- The assumption that women, and their partners, can and do make conscious decisions about pregnancy, childbearing and parenthood, including at the time of conception, underlies this three-way categorization. Much of the literature in developed countries demonstrates, however, that this usually far from reality. In developing countries, where personal volition and control over such planning and decisions are often much weaker, this assumption may well be invalid. Thus concepts of "intendedness", "wantedness" and the ability to control timing of these events are of little use analytically unless their meaning within a specific context is fully understood.

- Retrospective reporting of pregnancy desires can be influenced by the presence of the baby at the time of responding to questions, with an unintended pregnancy leading to a wanted baby. This implies that pregnancies that were unintended at the time of conception may be under-reported at the time of interview, and thus the actual proportion of unintended pregnancies is likely to be higher (Koenig et al 2006).

- Analyses of unintended pregnancy often aggregate unwanted with mistimed pregnancies, yet they usually reflect very different individual and social situations, with unwanted pregnancies more likely at the end of a woman's reproductive life and mistimed pregnancies more likely at the beginning although also likely to occur throughout her reproductive life (Luker 1999).

- Rapid changes in the practice and acceptability of premarital sex in many developing countries are changing the social context in which conception and first pregnancies occur. This may increase the possibility of an unintended pregnancy occurring, through 
more frequent but inconsistently protected sex, and of such pregnancies being considered mistimed rather than unwanted.

- The meaning of a mistimed pregnancy is not adequately captured in a dichotomous variable, i.e. "right" or "wrong" timing. A pregnancy that occurs several months before the preferred timing is likely to have different consequences for the woman and her partner than a pregnancy occurring a few weeks or months earlier than desired.

- DHS and other retrospective surveys usually collect information on unintended pregnancy from women only, and so little is known about the male partner's perceptions of pregnancy intendedness, or those of other family and friends that may influence the pregnancy's outcome. The literature that does include both partners' views, and the views of family members, has focussed mostly on attitudes towards family size ideals and use of contraception (Williams \& Sobieszczyk 2003).

- Quantitative and qualitative studies indicate tremendous ambivalence about contraception, conception, childbirth and parenthood that contribute to the many inconsistencies found between attitudes and behaviours. For example, some studies have found that substantial proportions of women becoming pregnant following contraceptive failure or who had an unmet need at the time of conception subsequently report a pregnancy as wanted (Bradley et al 2011; Ali et al 2012).

- Calculations of numbers of unintended pregnancy include all documented abortions on the assumption that only unintended pregnancies are terminated. However, this assumption does not allow for the possibility that a woman's circumstances may change after becoming pregnancy and so what may have been an intended pregnancy at the time of conception then becomes unwanted and is terminated.

"Although current measures of unintended pregnancy seem reasonable, reliable and predictive at a population level, they were not designed to be used at an individual level” (Santelli et al 2003: 99). Subsequent to the Santelli et al review, Tsui et al (2010) reviewed 21 individual-level, population-based, longitudinal or prospective multivariate analyses undertaken during the period following the previous review (2004-2009), of which five were in developing countries. Six studies examined the association between pregnancy intentions and birth outcomes and/or maternal health behaviours and outcomes; evidence of the effect of unintended pregnancies was inconclusive. Five studies (all US based) of pregnancy intentions and contraceptive use showed high proportions of women not using contraception despite stating intentions to defer or 
limit childbearing. Nine studies (five in developing countries) measured the incidence of unintended pregnancy following contraceptive use and found rates similar to published use-effectiveness rates.

\section{Measuring unintended pregnancy}

Measures based on retrospective responses to a cross-sectional survey questionnaire (i.e. the US NSFG and DHS approach) do seem to function fairly well when aggregated at the population level (Santelli et al 2003). However, using these measures to predict individual behaviour is problematic, as illustrated by the issues presented above. In response to the need for more valid and reliable measures, several efforts have been made to develop alternatives to the original version developed by the US NSFG (for examples of these, see listing in: Gipson et al 2008: Table A1; Tsui et al 2010: Tables 2\&3); some of these are described briefly.

Individual retrospective reports: The US NSFG uses a series of five questions to retrospectively measure pregnancy timing and desire (Santelli et al 2003:94-95). The DHS uses two standard questions to retrospectively measure the intention status of each birth at the time of its conception: (1) "At the time you became pregnant with (child's name), did you want to become pregnant then, did you want to wait until later, or did you not want to have any (more) children at all?" (2) "How much longer would you like to have waited before you became pregnant with (child's name)?" The second question permits identification of those births that occurred two or more years sooner than desired and so are considered mistimed. A pregnancy is then categorised as intended, unwanted or mistimed.

Multi-dimensional scaling: Santelli et al (2009) used disaggregated individual measures of pregnancy intention, attitude, timing and outcome in an exploratory factor analysis, which generated two broad dimensional scales, "mistiming of pregnancy" and "desire to become pregnant", which comprised both affective (happy to become pregnant) and cognitive (trying to become pregnant) elements. Two non-dimensional categories were also identified, "don't care" and "overdue". These measures were developed and validated using questions in the US NSFG. To be used in developing countries, however, would mean adding additional questions to DHS-type questionnaires and they could also be added to health and demographic surveillance systems. 
London Measure of Unintended Pregnancy (LMUP): Following two interviews with a cohort of women, once when pregnant and once afterwards, a conceptual model of pregnancy planning was developed that led to the creation of a six-item measure (contraceptive use, timing, intention, desire for a baby, partner discussion, and preconceptual preparations) to describe the circumstances of their pregnancy (Barrett \& Wellings 2002). Each item is scored 0-2, giving a continuous variable from 0 to 12 ; the instrument is used to measure retrospective perspectives. The LMUP has been validated in UK, US and India and is currently being validated in Malawi (Barrett et al 2004; Morof et al 2012; Rocca et al 2010; Hall et al 2013).

Prospective longitudinal studies: Two reviews of the literature (Santelli et al 2003; Tsui et al 2010) found four studies that followed cohorts over time to measure the association between contraceptive use, reproductive intentions and unintended pregnancy, two in the US and one each in Germany and Sri Lanka. Given the many advantages of prospectively measuring these variables, it is disappointing that more prospective studies have not been undertaken.

Population level measures of unwanted/excess fertility: The DHS uses women's responses to several questions to measure "unwanted fertility" as the proportion of women whose actual number of children is greater than the number they say they desire or consider ideal, i.e. the number of children born to a woman after her desired family size has been reached.

Population level data aggregation: The estimates developed by the Guttmacher Institute (Singh et al 2010) use population-level datasets to first calculate the number of all pregnancies by combining the number of live births, induced abortions and an estimated proportion of all miscarriages in a population. They then estimate unintended pregnancies by summing all unplanned births (using proportions derived from DHS-type surveys), all induced abortions (using standard incidence estimates applied to population estimates) and a proportion of all miscarriages (proportions of all miscarriages are allocated to intended and unintended pregnancies through a modelling exercise). Unintended pregnancies are measured as a proportion of all pregnancies and as an unintended pregnancy rate (UPR).

Modelling programme effectiveness from service statistics: Marie Stopes International has developed a model, called Impact 2, which uses service statistics to estimate the number of unintended pregnancies that do not happen because women are using family planning. It 
is based on method-specific failure rates and an assumption that if women were using no method there is a $40 \%$ likelihood of becoming pregnant (adjusted by age) (Weinberger et al 2012).

\section{Incidence of and trends in unintended pregnancy}

It is important to bear in mind that in the context of declining pregnancy rates worldwide (a 17 percent decrease in the number of pregnancies per 1,000 women in developed and developing countries between 1995 and 2008), rates of unintended pregnancy have generally decreased more than rates of intended pregnancy. This is especially the case in developed countries, where the unintended pregnancy rate decreased by 29 percent (due to rapidly increased access to effective contraception), whereas intended pregnancy decreased by two percent (Singh et al 2010: Table 2), reflecting the widespread achievement of fertility norms. In developing countries, the rates of unintended pregnancies over this period have also decreased overall (by 20\%), but there are marked differences between regions, with decreases of 25 percent in Latin America and 23 percent in Asia, but only seven percent in Africa, which highlights the regional differences in access to and use of effective contraception. Conversely, decreases in intended pregnancies in all of these regions has been much higher $(17 \%)$ than in developed countries, reflecting the on-going fertility transitions witnessed in most developed countries.

Singh et al (2010) have calculated that, in 2008, of approximately 208 million pregnancies worldwide, about 86 million pregnancies, or 41 percent, were considered unintended. There are, however, substantial variations in the proportions of unintended pregnancies by region (See Figure 1). The reasons for these regional variations are complex and represent interactions between desired family size, perceived and actual ability to practice family planning, availability and use of contraception and abortion, contraceptive use-effectiveness rates among other reasons. The tremendous variability in levels of and reasons for unintended pregnancy among different populations mean that it is essential to understand the underlying reasons for a pregnancy being considered unintended within the context of a particular population when designing policy or programming interventions. 
Figure 1: Proportions of total pregnancies that were reported as unintended, by region, 2008 (Singh et al 2010: Table 1)

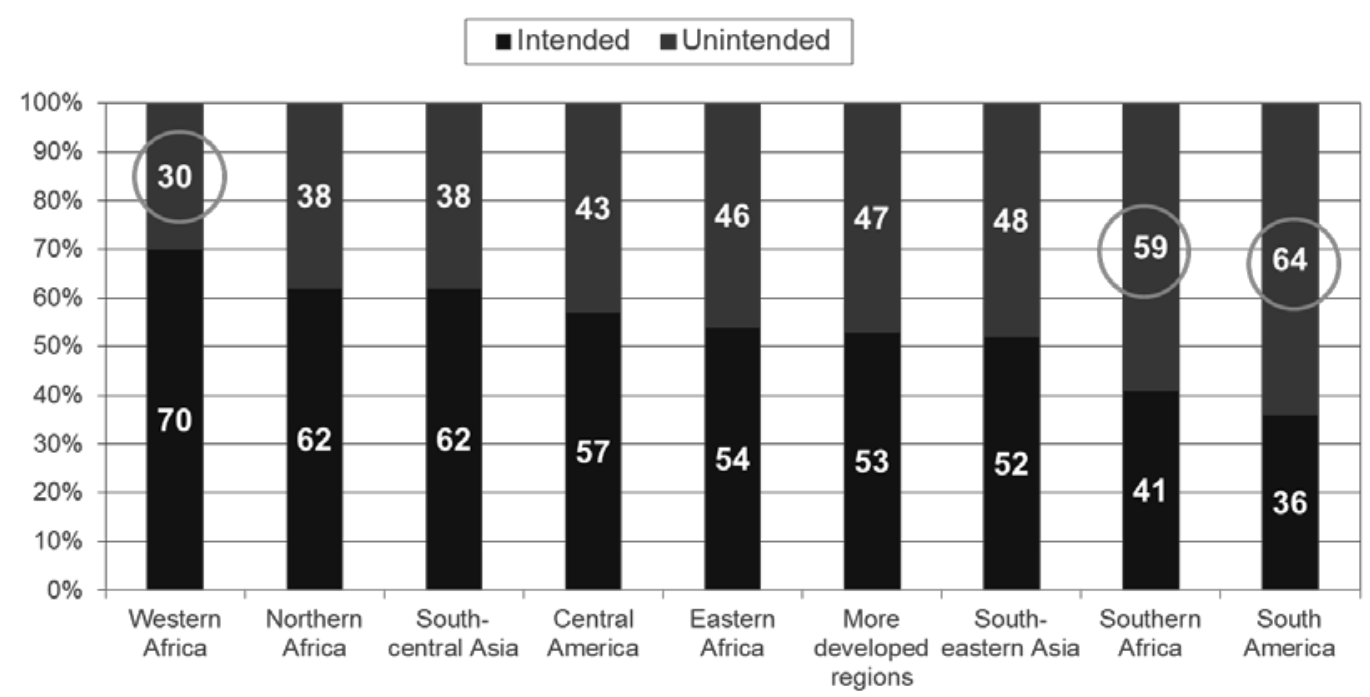

Policy statements advocate for reducing unintended pregnancies through increasing access to and use of FP services, on the perfectly reasonable assumption that the use of FP will increase the likelihood that a pregnancy can be intentionally planned. However, the proportions of pregnancies that are unintended (PPU) are often lower in countries with low contraceptive prevalence rates (CPR) and vice versa. Figure 1 demonstrates this at the regional level, with West Africa (PPU: 30\%; CPR: 9\%) and Latin America and the Caribbean (PPU: 64\%; CPR: 51\%) representing the extremes; developed countries, which have the highest CPR, are somewhere in between. Figure 2 presents this relationship using data from 158 DHS surveys for various developing countries and points in time, demonstrating consistency in the trend. 
Figure 2: Relation between national unintended pregnancy rates and modern contraceptive prevalence rates across 158 DHS, 1991-2007 (Tsui et al 2010: 164)

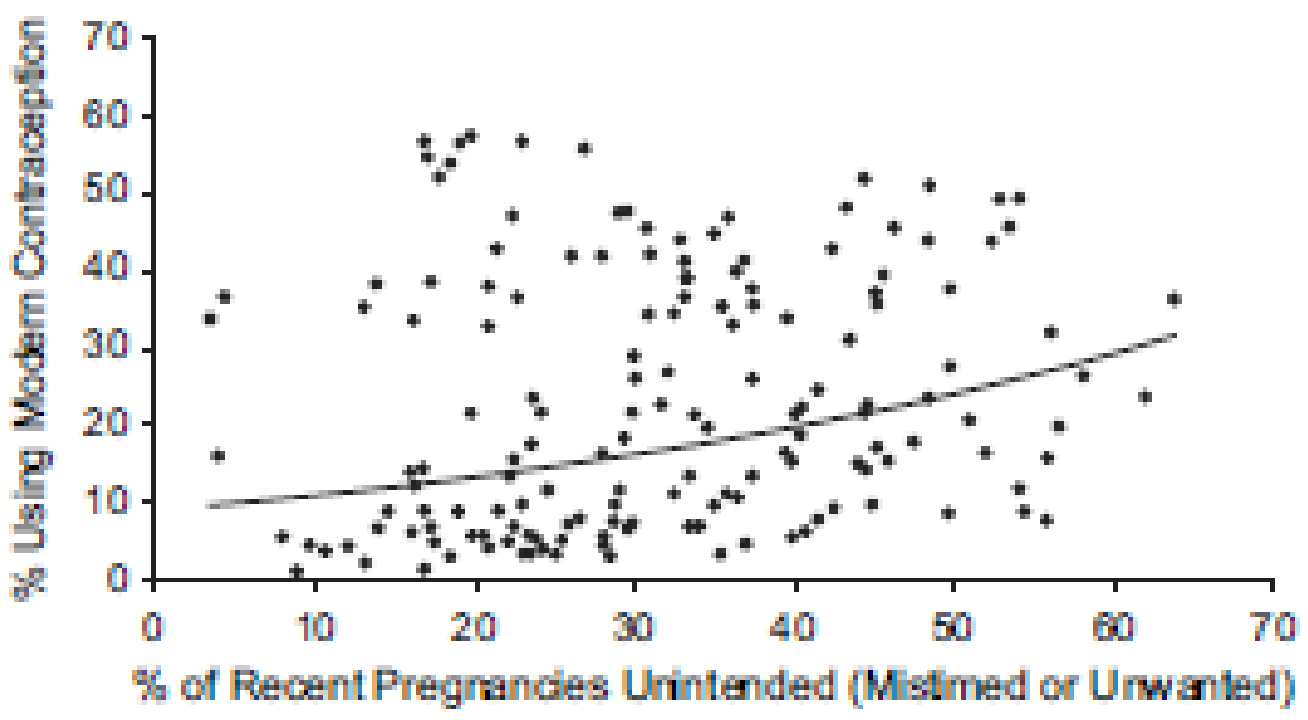

Figure 3 explores this relationship further by comparing regions in terms of the proportions of pregnancies that are unintended with the demand for family planning and with three indicators of programme performance: contraceptive prevalence, unmet need and proportion of demand satisfied (data were not easily available for South and Southeast Asia). Unintended pregnancy varies with demand and with CPR and demand satisfied, yet unmet need is fairly similar across the three regions shown. Thus, unintended pregnancy is high in countries where demand for family planning is high, the proportion of demand satisfied is high and contraceptive use is high - and vice versa. This analysis indicates that even where FP services are widely demanded and a substantial proportion of this demand is satisfied through women using contraception, up to two-thirds of women living in countries with high performing programs consider their last pregnancy to have been unintended. Whether unintended pregnancy at the population level can be reduced by increasing demand, reducing unmet need or increasing contraceptive prevalence is unclear; the policy and advocacy implications of these counter-intuitive associations are discussed below. 
Figure 3: Measures of pregnancy-related behaviours and programme performance, by region

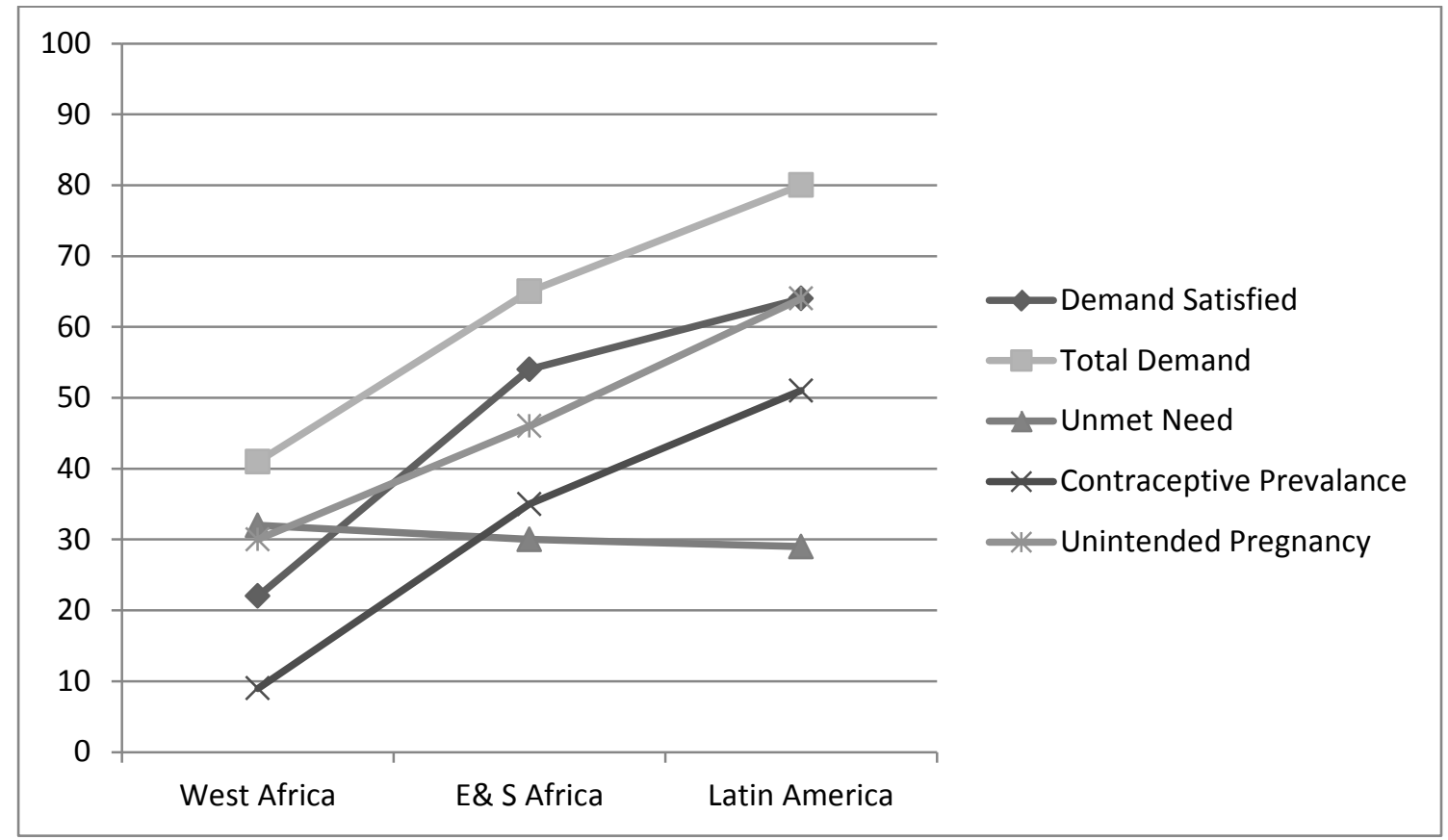

Analyses of the distribution of unintended pregnancies between populations within developing countries report that they are characterised by substantial differences (Gillespie et al 2007; Malarcher et al 2010), being much higher among the poor and unmarried adolescents. Malarcher et al remind us that unintended pregnancy is lower among married than unmarried adolescents because of personal and social expectations of becoming pregnant upon marriage, and so use of the concept of reducing unintended pregnancy as a policy goal for programming for adolescent populations needs to distinguish according to marital status; reducing unwanted pregnancies may be more appropriate among unmarried adolescents whereas reducing mistimed pregnancies may be more appropriate for married adolescents.

Whether these differences between populations should be considered inequalities or inequities is discussed by Gillespie and colleagues (2007). Defining an inequity as an inequality with moral implications that is considered unjust, they use DHS data to analyse unwanted fertility rates and CPR from 41 countries using the concentration index to quantify income-related inequalities within populations. Finding that unwanted births in the poorest quintile were more than twice that found in the wealthiest quintile, respectively 1.2 and 0.5 , although there was wide variation among the 41 countries, they caution that "that 
looking at family planning and fertility through an equity lens is justified for those countries with joint inequalities in unwanted fertility and access to family planning. In other countries, where there is little or no unwanted fertility inequity and where high fertility among the poor contributes to other health inequities, greater emphasis should be given to the health benefits of birth spacing and couples' rights to reproductive health information and services" (Gillespie et al 2007: 105).

Other variations in unintended pregnancies will be explored with data from four countries representing differing situations in terms of fertility desires, demand and its satisfaction, and use of contraception: Bangladesh, India, Kenya and Senegal. Figures 4 and 5 show the effect a strong FP program can have on unintended pregnancy; in 1992-3, Odisha State in northern India had a much higher UPR (41\%) than Senegal (28\%); by 2005 there was no change in Senegal but the proportion of pregnancies considered unintended was down to $18 \%$ in Odisha State. Another notable difference is that unwanted pregnancies are much higher than mistimed pregnancies, both absolutely and proportionately, in northern India than in Senegal, indicating differences in fertility desires or in perceived ability to control fertility, or both.

Figures $4 \& 5$ :Trends in mistimed and unwanted pregnancy over time

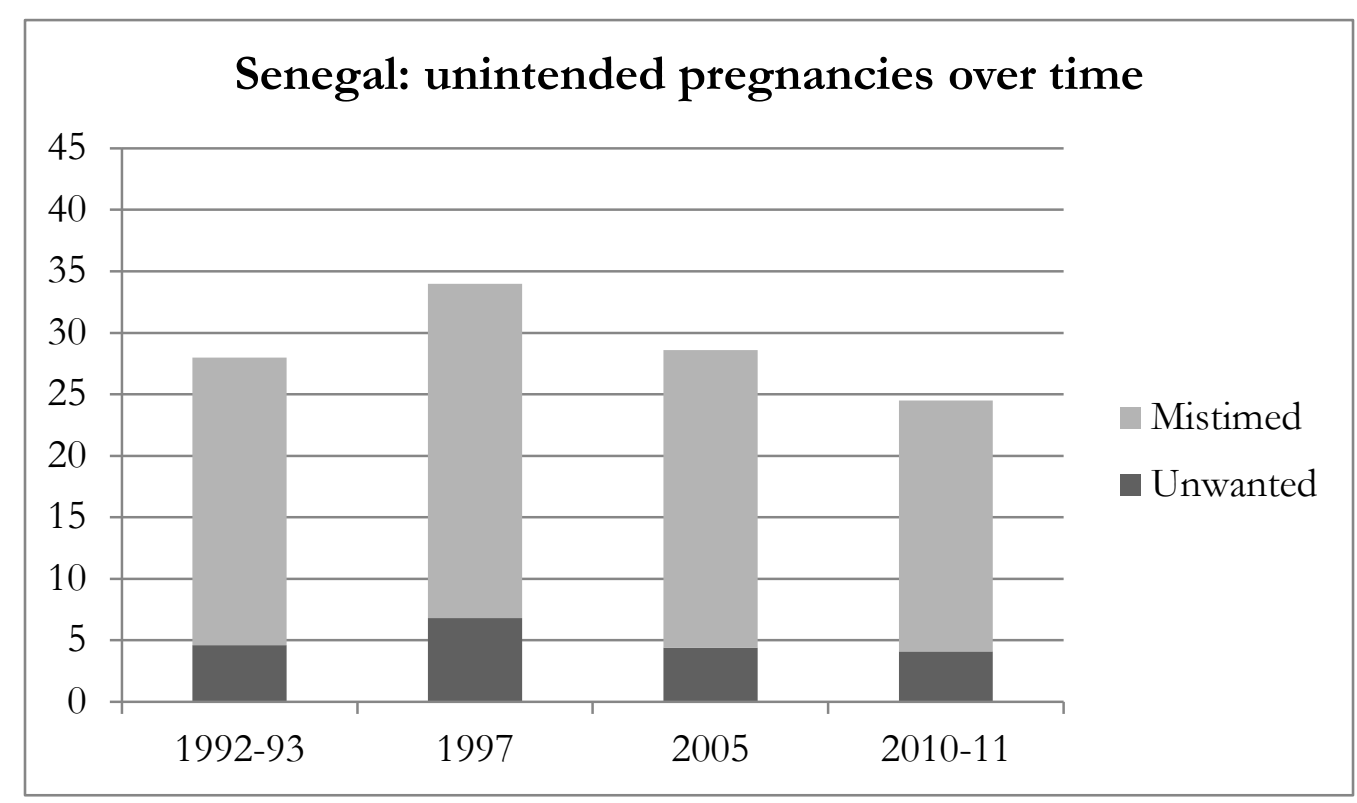




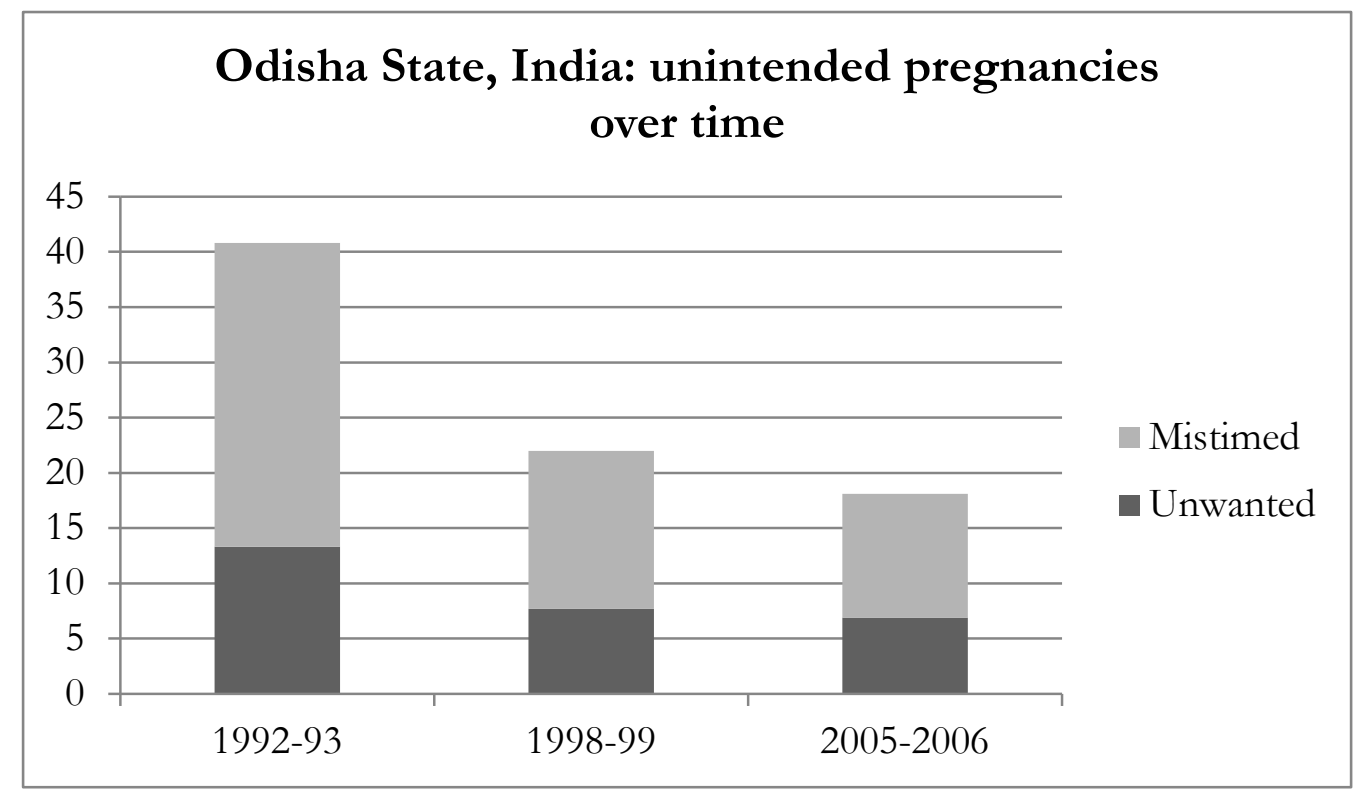

As Figure 6 shows, however, the global norm of mistimed pregnancies being more common than unwanted pregnancies is not always consistent across all populations; unintended pregnancies among the poorest in Bihar State, northern India, are much more likely to be unwanted than mistimed.

Figure 6: Proportions of mistimed and unwanted pregnancy by wealth group

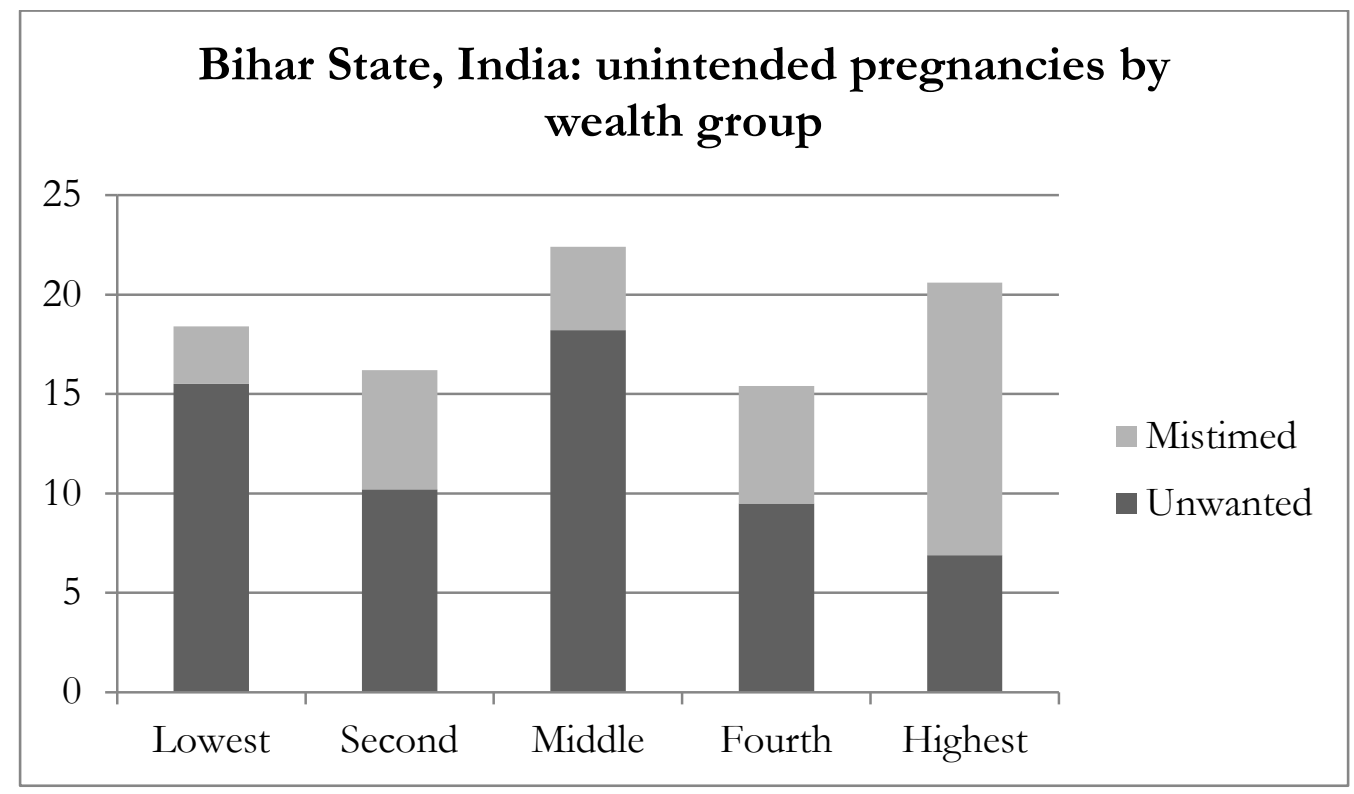


Figure 7 graphically describes the transition over the course of a woman's life cycle, using Bangladesh as an example - among younger women, unintended pregnancy is primarily mistimed whereas later in life they are primarily unwanted. Moreover, the likelihood of a pregnancy being unintended increases dramatically beyond the age of 30, highlighting the critical need for access to more effective contraceptive options among older women who have completed their desired family size.

Figure 7: Proportions of mistimed and unwanted pregnancy by age group

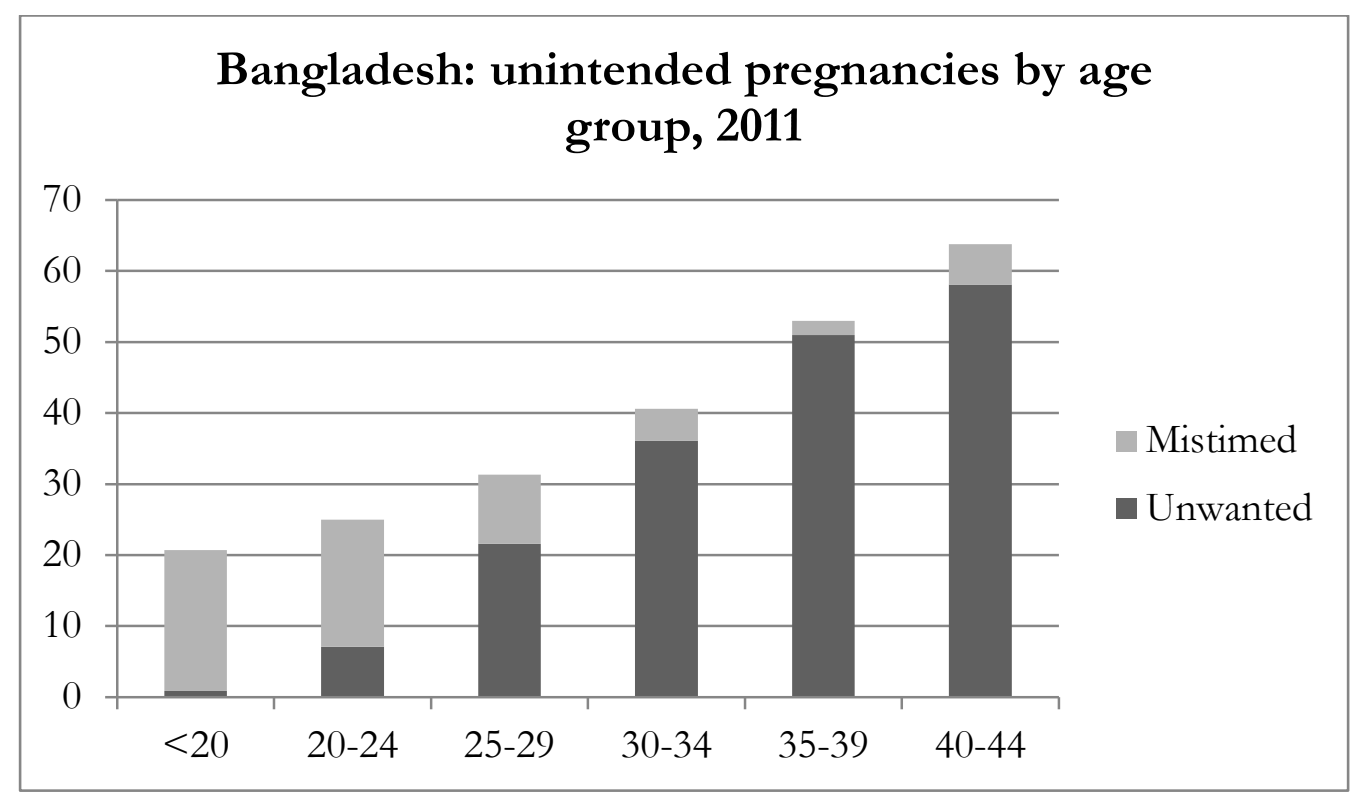




\section{Determinants and consequences unintended pregnancy}

For unintended pregnancy to function as a meaningful concept for advocacy statements as policy guidance and as a programming goal, it is helpful to understand the key determinants and consequences associated with unintended pregnancies, both immediate and distal.

Determinants of unintended pregnancy: Several factors determine most directly why unintended pregnancies occur. The majority of unintended pregnancies occur when a pregnancy is not planned or wanted and yet effective contraception is not being used, that is, when a woman has an unmet need for contraception. Singh and Darroch (2012) estimate that, on average, 79 percent of unintended pregnancies occur for this reason, i.e. because of an unmet need for effective methods. The reasons why a woman is not using contraception when she does not want to become pregnant, i.e. why she has an unmet need, vary considerably, remain poorly understood and are the subject of many conference papers, with most explanations relying on analyses of the reasons measured through questions asked by the DHS questionnaires (e.g. Sedgh et al 2007).

Although often presented in policy statements as reason why unintended pregnancies occur, unmet need does not account for one-fifth of unintended pregnancies that occur when a woman does not have an "unmet need". For example, 13 percent of unintended pregnancies occur to women using a traditional method (Singh and Darroch 2012); as these are considered less effective they are not included in the standard definitions of unmet need. Thus, if women who are using any method are included in the definition of unmet need, this reduces to 66 percent the proportion of women having an unintended pregnancy because of non-use of family planning.

Thus, on average, one-third of unintended pregnancies can occur when a woman is actively doing something to avoid becoming pregnant. For some women, a pregnancy may occur because of a "failure" in the effectiveness of the method being used, either because of the method's inherent inability to provide 100 percent prevention against pregnancy, or because the woman or couple are not able to use the method so that it can function to its full effectiveness. In practice, it is virtually impossible to separate whether failure occurred for one or other reason, although differences in rates have been estimated for 'consistent and correct use' (i.e. method failure) and 'as commonly used', albeit based largely on data from the US (WHO and CCP 2011: Appendix A). The proportion of pregnancies that occur following a failure ranges widely depending on characteristics of the user and of the 
method. Bradley et al (2011) analysed 20 DHS surveys and concluded that anywhere between five to 58 percent of unintended pregnancies occurred when women were using a contraceptive method. Failure rates at 12-months are lowest for permanent and long-acting reversible methods $(0-1.8 \%)$, followed by short-acting methods (average of $4.6 \%$ ), with rates when using traditional methods up to 22 percent.

An analysis of 60 DHS surveys in 24 countries by Ali and colleagues (Ali et al 2012) found that, for all methods and across all countries, 38 percent of women had discontinued using their method after 12 months. Furthermore, although this proportion is fairly similar (40 50 percent) among users of condoms, pills, injectables, periodic abstinence and withdrawal, for IUD users it was much lower at 13 percent (no data for implants presented). During this period, approximately six percent of women discontinued because they wanted to become pregnant and eight percent discontinued because they no longer needed to use a method (due to marital separation, cessation of sex or perceived inability to conceive); thus 24 percent of women discontinued because of method-related reasons or health concerns / side-effects or because of failure. For women using the pill, injectable or IUD, most discontinuation was for method-related or health concerns / side-effects, whereas for condom, periodic abstinence and withdrawal users it was mainly because of failure or a method-related reason - very few women using these methods discontinued because of health concerns or side-effects.

The proportion of women switching to another method after discontinuation ranges anywhere from 35-80 percent, with most women switching within three months and to a more effective, reversible method. By three months after discontinuation, $3-20$ percent of women had become pregnant and $12-74$ percent were at risk of pregnancy as they were not using any method. 
The analysis of DHS data from 20 countries by Bradley et al (2011) used the calendar method of data collection and modelled four scenarios to identify what would happen if contraceptive failure were to be reduced by users changing to more effective methods; this analysis assumes no increase in CPR (see Box 1). The results show that reductions in "unintended births", i.e. those resulting from an unintended pregnancy, would range from 10 percent to 27 percent depending on the type of switching practised. They conclude that their findings support the argument made by Anrudh Jain "that family planning programs should emphasize reducing unintended pregnancies among women who are already using contraception, rather than focusing exclusively on fulfilling unmet need among women who are not using contraception" (Jain 1999).

\section{Box 1: Effect of increasing use of more effective contraceptives on unintended pregnancy (Bradley et al 2011: ix-x)}

If all contraceptive failures were eliminated, unintended births would be reduced by nearly a third on average across countries. Induced abortion rates would be reduced by between one- and two-thirds. Though contraceptive failure would be difficult to eliminate entirely, we show that similar results could be achieved by the use of more effective contraceptive methods, without increasing total contraceptive prevalence rates, using 4 hypothetical scenarios:

1. All traditional method users switch to a short-term modern method.

2. Women using contraception for birth spacing switch to a short-term modern method, and women using contraception for limiting births switch to a long-acting modern method.

3. All non-long-acting and permanent method users switch to a reversible long-acting method of contraception.

4. All spacers switch to a long-acting method, while all limiters switch to a permanent method.

Scenario 1, in which all traditional method users switch to modern methods, would decrease unintended births by an average of 10 percent. Seventeen percent of unintended births could be prevented under Scenario 2, and more than a quarter of unintended births could be prevented under Scenario 3, in which all contraceptive users switch to long-acting methods. If all women who currently use contraceptives began using the most effective (long-acting or permanent) method to meet their fertility intentions, the level of unintended births would decrease by an average of 27 percent, varying from 4 to 54 percent across the countries analyzed. This scenario could prevent half a million unintended births each year in India, and between 4,000 and 241,000 unintended births per year in other countries. 
The wide range in country-specific frequencies and reasons for discontinuation, and in the three possible outcomes following discontinuation (pregnancy, switching another method, or non-protection (i.e. unmet need)) emphasizes the importance of population-specific analyses of the frequency, reasons and outcomes for discontinuation and switching. Understanding why women continue or not continue to use contraception is essential for identifying possible interventions that could facilitate rapid switching to an equally or more effective method for women not wanting to become pregnant and who are sexually active. As explained by Blanc et al (1999), without contraceptive failure or discontinuation, the total fertility rate would be one-third lower, and the unwanted fertility rate would be reduced by more than one-half, that is, it would be between 0.2 and 1.1 births lower.

Outcomes of pregnancy intendedness: An intended pregnancy usually has one of two immediate outcomes. Most result in a wanted birth but a small proportion end in a miscarriage; some intended pregnancies result in an induced abortion or unwanted birth if personal circumstances change following conception.

Unintended pregnancies can lead to one of three outcomes: i) a spontaneous abortion or miscarriage; ii) the pregnancy is terminated through an induced abortion (safely or unsafely); iii) a live birth, considered here to be an unplanned birth. The proportions of unintended pregnancies that result in unplanned births or in induced abortion vary widely, and these proportions are influenced by social norms, individual attitudes, and the legality and availability of safe abortion services. Singh et al (2010) estimate that, for all developing countries, of the 35 percent of pregnancies that are unintended (i.e. do not result in a miscarriage), 46 percent result in a live birth and 54 percent in an induced abortion. Unsurprisingly, the proportion resulting in an induced abortion tends to be higher in countries where abortion is legally available.

For unintended pregnancies that are terminated through induced abortion, the proportion that is undertaken safely depends primarily on the legality of abortion in a country, although even in countries where there are few legal restrictions, unsafe conditions may exist, particularly in countries in which abortion laws have recently been liberalized. WHO defines an unsafe abortion as "a procedure for termination of an unintended pregnancy done either by people lacking the necessary skills or in an environment that does not conform to minimum medical standards, or both" (WHO 2011). Illegal abortions are usually unsafe because: they are performed outside authorized facilities, sometimes in unsanitary conditions; the woman 
may not receive appropriate postabortion care; medical back-up unlikely to be immediately available should an emergency arise; and the woman might delay seeking an abortion or seeking care for complications because the abortion is clandestine. An analysis by Sedgh et al (2012) describes tremendous variation in the proportion of abortions that are performed unsafely: from 99.5 percent in West Africa to six percent in more developed countries.

Ahman and Shah (2011) estimate the rate of unsafe abortions per 1000 women aged 15 44 years and find a similarly wide range, from 36/1000 for eastern and middle Africa to $1 / 1000$ for developed countries. They also estimate the ratio of maternal deaths attributable to unsafe abortion, and find a similar distribution by region, from 100/100,000 live births in eastern and middle Africa to 1/100,000 live births in developed countries. However, they also show relatively low ratios of 8-10/100,000 in South and Central America, despite these regions having high rates of unsafe abortion (29-32/1000 women).

The relationships between unintended pregnancy, induced abortion and its association with morbidities and mortality are fairly well documented and understood; indeed, the need to prevent unintended pregnancy is often presented as a policy intervention to reduce the likelihood of women resorting to unsafe abortion and thereby increasing their risk of injury or death. Less well understood, are the relationships between unintended pregnancy, live births and the associated health care behaviours and health status of the mother and infant. While the majority of live births following an unintended pregnancy are considered mistimed or unwanted, in a study of six developing countries Curtis et al (2011: 63) "found that relatively high proportions of births were reported as intended following contraceptive failure or discontinuation for reasons other than wanting to get pregnant." This supports the evidence from developed countries discussed earlier that ambiguity may characterize many women and couple's thinking around conception and pregnancy.

Figure 8 presents a conceptual framework developed by Gipson and colleagues (2008) for describing the sequence of outcomes following pregnancy; it was used to guide a systematic review that analysed the role that pregnancy intendedness may have on four outcomes: maternal behaviour during pregnancy; birth outcomes; maternal postpartum behaviour; and infant and child health. 


\section{Figure 8: Potential effects of unintended pregnancy on infant, child, and parental health outcomes}

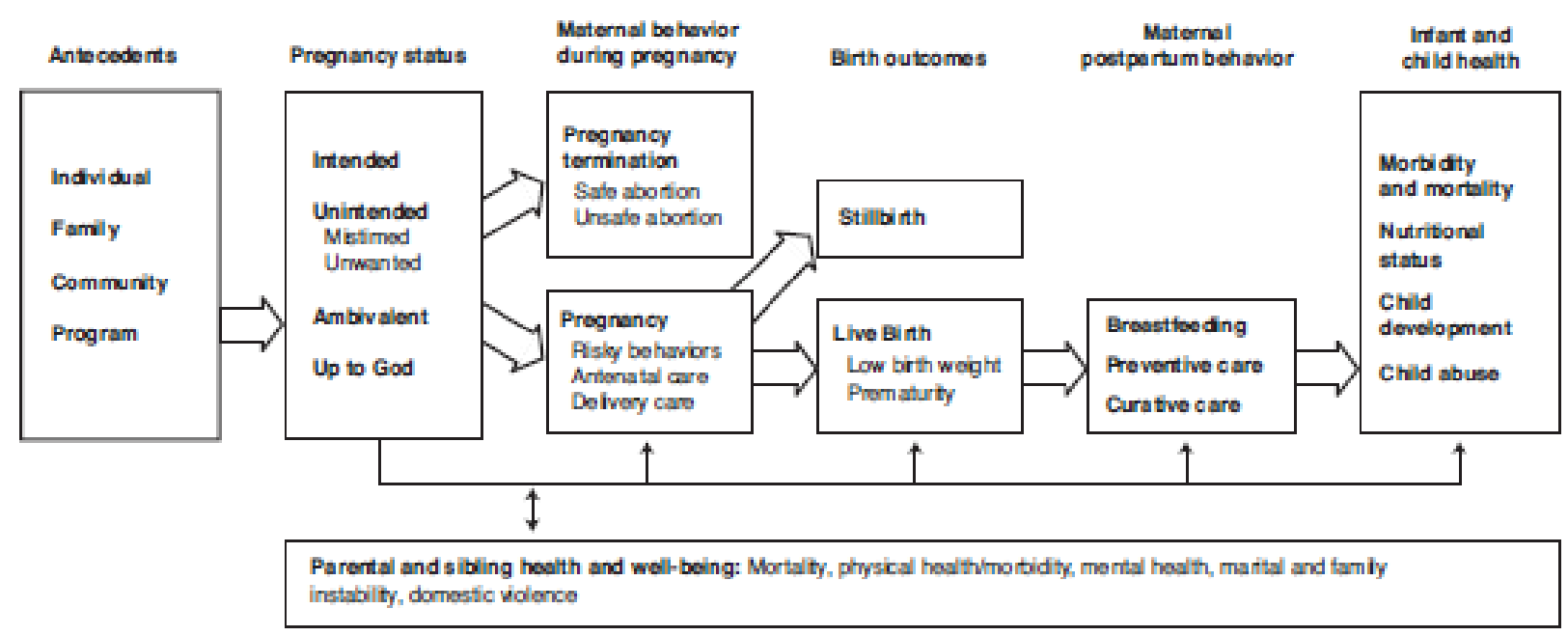

The review by Gipson and colleagues found that:

- Among studies that have assessed antenatal care, breastfeeding behaviour, and child nutrition, the evidence is relatively consistent, showing a negative effect of unintended pregnancy.

- For maternal risk behaviours, pregnancy outcomes, and curative care, developed country studies failed to find a significant association with pregnancy intention; the paucity of studies from developing countries precludes an overall assessment of such an impact. The few existing studies suggest that the children who result from unintended pregnancies may be disadvantaged with respect to low birth weight and incomplete vaccinations.

- Findings from rigorous studies in developing country suggest that children who are the result of unintended pregnancies are at an increased risk of infant mortality. Consistent evidence shows higher levels of mortality and malnutrition for female children as a result of son preference. Differentiation of the effects of being unintended versus the effect of the child's sex could help to broaden the understanding of differential treatment and underinvestment in children.

- Relatively few studies have assessed the effects of unintended pregnancy on women's health and well-being. The studies that have been conducted indicate a positive association between unintended pregnancy and depression, anxiety, and abuse. 
- Research is needed to assess whether unintended pregnancy results in adverse physical and mental health outcomes for both men and women.

- There is very limited research on older children, adolescents and adults born of unintended pregnancies, primarily because of the need for longitudinal designs over many years or generations. The few studies that do exist (for example, Henry David's study of children born to women denied an abortion (David 2006)) suggest that there may well be long-term and cumulative effects on health and development during and beyond childhood.

Malarcher and colleagues (2010) have summarized the salient issues associated with unwanted childbearing (see Box 2). They concur with Gipson et al (2008) in emphasizing the surprising paucity of research on the longer-term outcomes of unplanned births given the prominence of unintended pregnancy as a policy and investment goal.

\section{Box 2: Unwanted childbearing (Malarcher et al 2010: 183)}

Unwanted childbearing detrimentally affects women and children. Women who have an unwanted pregnancy are more likely to delay antenatal care or have fewer visits. Unwanted children are more likely to experience symptoms of illness, such as acute respiratory infection and diarrhoea, less likely to receive treatment or preventive care such as vaccinations, less likely to be breastfed and more likely to have lower nutritional status, and have fewer educational and development opportunities. A recent review concluded that "children who are the result of unintended pregnancies are at an increased risk of infant mortality compared with children resulting from intended pregnancies".

Unwanted childbearing negatively influences the mother-child relationship and maternal health. Unintended pregnancy is associated with maternal depression, anxiety and abuse. Unintended childbearing among adolescents is particularly detrimental, increasing vulnerability by truncating educational opportunities, increasing welfare dependence and increasing the probability of domestic violence.

Women with fewer social and financial assets may view unintended childbearing as less problematic than women with opportunities outside the home. Women faced with poor economic conditions, low self-esteem and lack of moral support may see motherhood as a means of escape. 


\section{Summary and conclusions}

Unintended pregnancy is considered an undesirable condition, both in terms of the personal effects on a woman, her partner and family and the resulting child, and on the population-level outcomes of unwanted fertility and mortality and morbidity associated with unsafe abortion when used to manage such pregnancies. This paper discusses the meaning and measurement of unintended pregnancy, showing its fluid conceptualization and that there is no single or straightforward means by which it can and should be measured. Some examples of how the experience and burden of unintended pregnancy is distributed across populations are given, which again highlight the tremendous variability and the need to carefully analyse how and why it occurs within particular populations before identifying investment strategies to reduce it.

Despite the apparent definitiveness of the concept, individual reported experiences of unintended pregnancy are characterized by high levels of ambiguity, and so any measures must be interpreted accordingly. However, when aggregated to population levels, such measures do appear to be fairly valid and reliable, although retrospective measures are likely to under-estimate the true frequency. Efforts to develop multi-dimensional measures that better describe what is clearly a multi-faceted attitude are promising and would benefit from greater attention, particularly given the sustained importance of reducing and preventing unintended pregnancy as a policy and programming goal. Although the associations between unintended pregnancy and induced abortion are well understood and clearly articulated, the outcomes associated with births following an unintended pregnancy are less well-researched and represent a major evidence gap. Such evidence is important and research should be prioritized because what is known indicates that adverse maternal, new-born and child health outcomes following an unintended pregnancy are usually more severe than for an intended pregnancy. Moreover, adverse outcomes associated with infant, child and young adult development for a birth following a denied abortion may be particularly severe and have consequent additional costs for health, social and educational services.

Part of the attractiveness of unintended pregnancy as a policy goal is that it can be interpreted as a rights-based indicator for FP programming that is neither explicitly demographic- or health-oriented. Becoming pregnant only when intended is a basic reproductive right ("children by choice, not by chance") and so a reduction in unintended 
pregnancies demonstrates progress towards improving women's reproductive rights more explicitly than efforts to reduce their unmet need for or use of contraception. Moreover, the concept of unintended pregnancy can embrace both 'prevention' through consistent use of effective contraception and 'management' through access to and use of safe abortion. This duality is usually reflected in policy terminology that justifies reducing unintended pregnancy as a means to reduce the need for abortion, and particularly unsafe abortion. A more comprehensive rights-based interpretation may also include a policy imperative to enable access to safe abortion to prevent an unplanned birth in addition to reducing the need for abortion.

Unintended pregnancies can be reduced by increasing access to FP, thereby reducing unmet need for contraception. However, an investment only in reducing unmet need would ignore the two-thirds of women who have an unintended pregnancy while using some form of family planning. Several analysts have demonstrated the tremendous reductions in unintended pregnancy that could be achieved simply by increasing the effectiveness with which current users practice family planning. Thus a combined policy strategy that invests in both reducing unmet need and improves the effectiveness of contraceptive technologies and their consistent use is essential for reducing unintended pregnancy.

Because of the ambiguities associated with personal experiences with unintended pregnancy, and because it can be reduced by both increases in the use of contraception and in the effectiveness with which contraception is practised, the population-level relationships between unintended pregnancy and the demand for and use of contraception are somewhat counter-intuitive. At the regional level, the lowest proportions of unintended pregnancy occur in countries with low CPR and low proportions of demand satisfied, and not in countries with high use of contraception and demand satisfied. As these are also the countries in which demand for family planning is also low, this reflects preferences for high wanted fertility and so unintended pregnancies, especially mistimed pregnancies, are likely to be lower at the individual and population levels. Interestingly, unmet need does not appear to be associated with unintended pregnancy - and indeed is generally lower when unintended pregnancy is higher - and so recommendations to reduce unintended pregnancy through reducing unmet need should be made cautiously. 
The concept of unintended pregnancy could be more fully exploited as a policy imperative, however. First, it could be used to highlight that the right to not have an unintended pregnancy be accompanied by the right to have an intended pregnancy. There would appear to a missed opportunity to advocate for and invest in programming to address the rights of those experiencing infertility or subfertility to have access to services that would enable achievement of an intended pregnancy. Secondly, almost half of all unintended pregnancies result in an unplanned birth. The available evidence suggests that the increased likelihood of adverse health, psychological and social development outcomes associated with the infants and children born of unintended pregnancies, including those born after an abortion was denied, may require substantial expenditures by the state, couple and family. Further understanding of these consequences and their costs would greatly benefit government investments in infant and child support and would further strengthen advocacy to support investments in reducing unintended pregnancy.

Unintended pregnancy is usually presented in policy statements as having a direct and linear relationship with unmet need. As demonstrated here, this is frequently not the case. While partially attributable to its definition and measurement, a broader understanding of the determinants and consequences would improve both policy guidance and programming investments. 


\section{References}

Ahman E \& Shah 1. 2011. "New estimates and trends regarding unsafe abortion mortality", International Journal of Gynecology and Obstetrics, 115(2): 121-126.

Ali M., Cleland J \& Shah I. 2012. Causes and Consequences of Contraceptive Discontinuation: Evidence from 60 Demographic and Health Surveys. WHO: Geneva.

Barrett G \& Wellings K. 2002. "What is a 'planned' pregnancy? empirical data from a British study", Social Science and Medicine, 55: 545-557.

Barrett G, Smith S \& Wellings K.2004. "Conceptualisation, development, and evaluation of a measure of unplanned pregnancy", Journal of Epidemiology and Community Health. 58:426433.

Blanc, A, Curtis S,\& Croft T. 1999. Does Contraceptive Discontinuation Matter? Quality of Care and Fertility Consequences. MEASURE Evaluation Technical Report Series No. 3. Carolina Population Center: Chapel Hill.

Bradley S, Croft T, \& Rutstein S. 2011. The Impact of Contraceptive Failure on Unintended Births and Induced Abortions: Estimates and Strategies for Reduction. DHS Analytical Studies No. 22. ICF Macro: Calverton.

Cu Le L, Magnani R, Rice J, Speizer I \& Bertrand W. 2004. "Reassessing the level of unintended pregnancy and its correlates in Vietnam" Studies in Family Planning, 35(1): 15-26.

Curtis S, Evens E \& Sambisa W. 2011. "Contraceptive discontinuation and unintended pregnancy: An imperfect relationship", International Perspectives on Sexual and Reproductive Health, 37(2):58-66.

David H. 2006. "Born unwanted, 35 years later: The Prague Study", Reproductive Health Matters,14(27): 181-190.

DFID. 2010. Choices for Women: Planned Pregnancies, Safe births and Healthy Newborns. DFID, London.

Exavery A, et al. 2013. "How mistimed and unwanted pregnancies affect timing of antenatal care initiation in three districts in Tanzania", BMC Pregnancy and Childbirth, 13:35.

Gillespie D, Ahmed S, Tsui A \& Radloff S. 2007. Unwanted fertility among the poor: an inequity? Bulletin of the World Health Organization, 85(2):100-107.

Gipson J, Koenig M \& Hindin M. 2008. "The effects of unintended pregnancy on infant, child, and parental health: A review of the literature", Studies in Family Planning, 39(1):18-38.

Hall J, Barrett G, Malata A \& Stephenson J. 2013. "Improving the measurement of unintended pregnancy to improve reproductive health", First Global Conference on Contraception, Reproductive and Sexual Health, May 22- 25, Copenhagen, Denmark.

ICPD. 1994. Programme of Action of the International Conference on Population and Development. United Nations, New York.

Jain, A. 1999. "Should eliminating unmet need for contraception continue to be a program priority?”, International Family Planning Perspectives 25(Supplement):S39-S43 \& S49.

Koenig M, Acharya R, Singh S \& Roy T. 2006. "Do current measurement approaches underestimate levels of unwanted childbearing? Evidence from rural India", Population Studies, 60(3): 243-256. 
Luker K. 1999. "A reminder that human behavior frequently refuses to conform to models created by researchers". Family Planning Perspectives 31(5): 248-249.

Malarcher, S, Olson L \& Hearst N. 2010. "Unintended pregnancy and pregnancy outcome: equity and social determinants". Equity, Social Determinants and Public Health Programmes, edited by E. Blas and A. Kurup. WHO: Geneva.

Marston C \& Cleland J. 2003. "Do unintended pregnancies carried to term lead to adverse outcomes for mother and child? An assessment in five developing countries", Population Studies, 57(1):77-93.

Morof D, Steinauer J, Haider S, Liu S, Darney P, et al. 2012. "Evaluation of the London Measure of Unplanned Pregnancy in a United States Population of Women" PLoS ONE 7(4): e35381.

Rocca CH, Krishnan S, Barrett G, Wilson M. 2010. "Measuring pregnancy planning: An assessment of the London Measure of Unplanned Pregnancy among urban, south Indian women" Demographic Research, 23: 293-334.

Santelli J, Rochat R, Hatfield-Timajchy K, Colley Gilbert B, Curtis K, Cabral R, Hirsch J, Schieve L and Unintended Pregnancy Working Group. 2003. "The measurement and meaning of unintended pregnancy". Perspectives on Sexual and Reproductive Health 35(2): 94101.

Santelli J, Lindberg L, Orr M, et al. 2009. "Toward a multidimensional measure of pregnancy intentions: evidence from the United States". Studies in Family Planning. 40(2):87100.

Sedgh G, Hussain R, Bankole A \& Singh S. 2007. Women with an unmet need for contraception in developing countries and their reasons for not using a method, Occasional Report No. 37.

Guttmacher Institute: New York.

Singh S \& Darroch JE. 2012. Adding It Up: Costs and Benefits of Contraceptive Services-Estimates for 2012. Guttmacher Institute and UNFPA: New York.

Singh S, Sedgh G \& Hussain R. 2010. "Unintended pregnancy: Worldwide levels, trends, and outcomes". Studies in Family Planning. 41(4): 241-250).

Stephenson R, Koenig M, Acharya R \& Roy. T.2008. "Domestic violence, contraceptive use, and unwanted pregnancy in rural India", Studies in Family Planning, 39(30): 177-186.

Tsui A, McDonald-Mosley R \& Burke A. 2010. "Family planning and the burden of unintended pregnancies". Epidemiologic Reviews. 32:152-174.

UNFPA. 2012. By choice, not by chance: Family planning, human rights and development, UNFPA. New York.

USAID. 2011. USAID Policy Framework 2011 - 2015. USAID, Washington.

Weinberger M, Pozo-Martin F, Boler T, Fry K \& Hopkins K. 2012. Impact 2: An innovative tool for estimating the impact of reproductive health programmes Methodology paper, Marie Stopes International: London.

Westoff C. 2012. Unmet Need for Modern Contraceptive Methods. DHS Analytical Studies No. 28. ICF International: Calverton.

Williams L \& Sobieszczyk T. 2003. "Couple attitudes and agreement regarding pregnancy wantedness in the Philippines", Journal of Marriage and Family, 65(4): 1019-1029. 
WHO. 2004. Reproductive Health Strategy. WHO: Geneva.

WHO. 2011. Unsafe abortion: global and regional estimates of the incidence of unsafe abortion and associated mortality in 2008, 6th edn. WHO: Geneva.

WHO and CCP. 2011. Knowledge for Health Project. Family Planning: A Global Handbook for Providers. CCP and WHO: Baltimore and Geneva. 